\title{
THE STRUCTURE OF ULTRAPRODUCTS OF ABELIAN GROUPS
}

\author{
PAUL C. EkLOF
}

\begin{abstract}
A structure theorem is given which characterizes abelian groups which are ultrapowers with respect to $\omega$-incomplete ultrafilters. It is also proved that any nonprincipal ultraproduct of abelian groups over a countable index set-or, more generally, with respect to a good ultrafilter-is an ultrapower with respect to an $\omega$-incomplete ultrafilter. The results of this paper provide a solution to a problem of $L$. Fuchs.
\end{abstract}

1. Preliminaries on ultraproducts. We begin by recalling some basic facts and definitions. (For further information, useful references are [1], [2], and [9].) Let $\kappa$ be an infinite cardinal; we identify $\kappa$ with the set of ordinals less than $\kappa: \kappa=\{\nu: \nu<\kappa\}$. We write $\omega_{\nu}$ for the $\nu$ th infinite cardinal; in particular, $\omega=\omega_{0}=$ the set of finite ordinals. Throughout this section $D$ denotes a nonprincipal ultrafilter on $\kappa$ i.e., $D$ is a set of subsets of $\kappa$ satisfying:

(a) $\varnothing \in D$;

(b) $S, T \in D \Rightarrow S \cap T \in D$;

(c) $S \in D, S \subseteq T \subseteq \kappa \Rightarrow T \in D$;

(d) $S \subseteq \kappa \Rightarrow S \in D$ or $\kappa-S \in D$;

(e) $F \leqq \kappa, F$ finite $\Rightarrow \kappa-F \in D$.

If $\left\{A_{\nu}: \nu<\kappa\right\}$ is a set of sets indexed by $\kappa$, the ultraproduct of the $A_{\nu}$ with respect to $D$, denoted $\Pi_{\nu<\kappa} A_{\nu} / D$ is the quotient of $\Pi_{\nu<x} A_{\nu}$ by the equivalence relation

$$
f \equiv{ }_{D} g \text { iff }\{\nu: f(\nu)=g(\nu)\} \in D
$$

for any $f, g \in \prod_{\nu<x} A_{\nu}$. We denote the equivalence class of $f \in \Pi_{\nu<x} A_{\nu}$ by $f / D$. If the $A_{\nu}$ are abelian groups, we make $\Pi_{\nu<r} A_{\nu} / D$ into a group by defining

$$
f / D+g / D=(f+g) / D .
$$

If $A_{\nu}=A$ for all $\nu<\kappa$, we denote the ultraproduct by $A^{\kappa} / D$ and call it the ultrapower of $A$ with respect to $D$.

It is well-known that if $D$ does not satisfy (e)-i.e., if $D$ is principal-then there exists $\nu_{0}<\kappa$ such that the map

$$
\prod_{\nu<\kappa} A_{\nu} / D \longrightarrow A_{\nu_{0}}: f / D \longmapsto f\left(\nu_{0}\right)
$$

is an isomorphism. Thus every group is an ultraproduct with respect to a principal ultrafilter; for this reason we confine our attention to 
nonprincipal ultrafilters.

We shall be interested in the cardinality of ultraproducts; in stating results about cardinality we will identify an ultraproduct of cardinals with its cardinal number and write $\beta=\prod_{\nu<k} \alpha_{\nu} / D$ for $\beta=$ Card $\left(\Pi_{\nu<\kappa} \alpha_{\nu} / D\right)$, when the $\alpha_{\nu}$ are cardinals. The simplest result we can state is the following (see [1], Chapter 6, Lemma 3.6).

Lemma 1.1. If $\alpha$ is a finite cardinal, then $\alpha=\alpha^{\kappa} / D$.

If $\alpha$ is infinite, it is much harder to determine the cardinality of $\alpha^{\kappa} / D$, and we shall have to impose some additional restrictions on $D$. We say $D$ is $\omega$-incomplete if there exists sets $Y_{n} \notin D$ such that $Y_{n} \cap$ $Y_{m}=\varnothing$ for $n \neq m$ and $\mathrm{U}_{n<\omega} Y_{n}=D$.

Lemma 1.2. (Keisler [8].) Let $D$ be $\omega$-incomplete and for each $\nu<\kappa$ let $\alpha_{\nu}$ be an infinite cardinal. Then

$$
\left(\prod_{\nu<\kappa} \alpha_{\nu} / D\right)^{\omega}=\prod_{\nu<\kappa} \alpha_{\nu} / D .
$$

Lemma 1.3. (Frayne-Morel-Scott [4]). If $D$ is an $\omega$-incomplete ultrafilter on $\kappa$, and $\left\{\alpha_{\nu}: \nu<\kappa\right\}$ is a sequence of cardinals such that for all finite cardinals $m,\left\{\nu: \alpha_{\nu}<m\right\} \notin D$ then

$$
\prod_{\nu<\kappa} \alpha_{\nu} / D \geqq 2^{\aleph_{0}} .
$$

We get even better results for regular ultrafilters. $D$ is called $(\omega, \lambda)$-regular if there is a function $f$ mapping $\kappa$ into the finite subsets of $\lambda$ such that for all $\nu<\lambda,\{\mu<\kappa: \nu \in f(\mu)\} \in D$. We say of such an $f$ that it makes $D(\omega, \lambda)$-regular. $D$ is called regular if it is $(\omega, \kappa)$ regular. It is easily seen that $D$ is $(\omega, \omega)$-regular if and only if $D$ is $\omega$-incomplete.

Lemma 1.4. (Keisler [8].) Suppose $f$ makes $D(\omega, \lambda)$-regular; let $m_{\nu}=$ cardinality of $f(\nu)$. Then for any cardinals $\alpha_{\nu}, \nu<\kappa$,

$$
\prod_{\nu<\kappa} \alpha_{\nu}^{m_{\nu}} / D \geqq\left(\prod_{\nu<\kappa} \alpha_{\nu} / D\right)^{\lambda} .
$$

In particular, if all the $\alpha_{\nu}$ are infinite,

$$
\prod_{\nu<\kappa} \alpha_{\nu} / D=\left(\prod_{\nu<\kappa} \alpha_{\nu} / D\right)^{\lambda} .
$$

CoRollary 1.5. (Frayne-Morel-Scott [4].) If $D$ is regular and $\alpha$ is an infinite cardinal, then $\alpha^{\kappa} / D=\alpha^{\kappa}$.

COROLLARY 1.6. Let $D$ be regular and let $\left\{\alpha_{\nu}: \nu<\kappa\right\}$ be a set of 
infinite cardinals and suppose $\gamma=$ least cardinal such that $\left\{\nu: \alpha_{\nu}>\right.$ r\} $\notin D$. Then $\Pi_{\nu<x} \alpha_{\nu} / D=\gamma^{k}$.

Proof. If $\gamma$ is a successor cardinal, the result follows from Lemma 1.5. So assume $\gamma$ is a limit cardinal and let $\lambda=\prod_{\nu<x} \alpha_{\nu} / D$; clearly $\lambda \leqq \gamma^{\kappa}$. If $\lambda \geqq \gamma$, then by $1.4, \lambda=\lambda^{\kappa} \geqq \gamma^{k}$ and we are done. Otherwise, there exists a cardinal $\rho$ such that $\lambda<\rho<\gamma$; by definition of $\gamma,\left\{\nu: \alpha_{\nu}>\rho\right\} \in D$, so $\lambda \geqq \rho^{\kappa} / D=\rho^{\kappa} \geqq \rho>\lambda$, a contradiction.

How much of a loss of generality do we suffer in the answer to Problem 25 of [5] by restricting ourselves to $\omega$-incomplete or regular ultrafilters? For countable index sets the answer is that there is no loss; indeed an ultrafilter on $\omega$ is nonprincipal $\Leftrightarrow$ it is $\omega$-incomplete $\Leftrightarrow$ it is regular ([1], Chapter 6, Lemma 1.17). For uncountable index sets the answer is connected with open problems in set theory, as follows.

It is consistent with Zermelo-Frankel set theory plus the axiom of choice $(Z F+A C)$ to assume that every nonprincipal ultrafilter on any index set is $\omega$-incomplete. It is not known whether this assumption is a theorem of $Z F+A C$; however, it can be proved that if there is $\kappa$ such that there exists a nonprincipal $\omega$-complete ultrafilter on $\kappa-$ in which case, we say $\kappa$ is $\omega$-measurable - then $\kappa$ is extraordinarily large (e.g. $\kappa$ is inaccessible and there are $\kappa$ inaccessibles cardinals $<\kappa$-see [15] for more details). Moreover, it can be proved that if there exists an $\omega$-measurable cardinal and $\kappa$ is the smallest one, then any group of cardinality $<\kappa$ is an ultrapower; in fact, any $\omega$-complete ultrafilter $D$ on $\kappa$ is $\lambda$-complete for all $\lambda<\kappa$ ([1], Chapter 6, Theorem 1.11), from which it follows easily that if $\operatorname{Card}(A)<\kappa$, then $A$ is isomorphic to $A^{\kappa} / D$.

A regular ultrafilter is $\omega$-incomplete and also uniform. ( $D$ is uniform means for every $S \in D$, Card $(S)=\kappa$.) Every ultraproduct with respect to a nonprincipal ultrafilter is an ultraproduct with respect to a uniform ultrafilter ([1], Chapter 6, Corollary 2.4), so there is no loss of generality for our purposes in restricting attention to uniform ultrafilters. It is an open problem whether every $\omega$-incomplete uniform ultrafilter on $\kappa$ is regular. However, it has been proved (by Prikry [13] for $n=1$ and Jensen [6] for arbitrary finite $n$ ) that it is consistent with $Z F+A C$ to assume that for any finite $n$ every uniform ultrafilter on $\omega_{n}$ is regular. (More precisely, this is true in the constructible universe.)

In $\S 3$ we shall need the following result on cardinalities of ultraproducts. We make use of the following terminology. We say that a property $P$ holds almost everywhere (a.e.) with respect to $D$ in $\left\{A_{\nu}\right.$ : $\nu<\kappa\}$ iff $\left\{\nu<\kappa: P\right.$ holds in $\left.A_{\nu}\right\}$ is an element of $D$. For example, Card $A_{\nu}=\gamma$ a.e. (w.r.t. $D$ ) iff $\left\{\nu<\kappa\right.$ : Card $\left.A_{\nu}=\gamma\right\} \in D$. As another 
example of this terminology, we say that a function $f: \kappa \rightarrow \omega$ is a.e. constant (w.r.t. $D$ ) if $\exists n<\omega$ such that $\{\nu<\kappa: f(\nu)=n\} \in D$.

THEOREM 1.7. Let $D$ be a nonprincipal ultrafilter on $\omega$. Suppose that for each $k \in \omega,\left\{\alpha_{n, k}: n<\omega\right\}$ is a nonincreasing sequence of cardinals. For each $n<\omega$ let $\beta_{n}=\prod_{k<\omega} \alpha_{n, k} / D$; and let $\beta=\lim _{n \rightarrow \infty} \beta_{n}$. Then there exists $l: \omega \rightarrow \omega$ such that $l$ is not a.e. constant (w.r.t. $D$ ) and $\Pi_{k<\omega} \alpha_{l(k), k} / D=\beta$.

Proof. Since $\beta=\beta_{n}$ for sufficiently large $n$, we may as well suppose that $\beta=\beta_{n}$ for all $n$. If $\beta$ is finite, then for each $n, \alpha_{n, k}=$ $\beta$ a.e. (w.r.t. $D$ ) and hence we can define $l(k)=$ largest $n \leqq k$ such that $\alpha_{n, k}=\beta$. Therefore, we may suppose $\beta$ is infinite, and so by Lemma $1.2, \beta^{\omega}=\beta$. Let $\gamma$ be the smallest infinite cardinal such that $\gamma^{\omega}=\beta$. We consider two cases:

Case 1. The cofinality of $\gamma>\omega$. Define $l: \omega \rightarrow \omega$ by $l(k)=$ largest $n \leqq k$ such that $\alpha_{n, k} \geqq \gamma$, or $l(k)=0$ if no such $n$ exists. Then $l$ is not a.e. constant (w.r.t. $D$ ), because otherwise there exists $n<\omega$ and $S \in D$ such that for all $k \in S, \alpha_{n+1, k}<\gamma$; since $c f(\gamma)>\omega$, there exists $\delta<\gamma$ such that $\alpha_{n+1, k} \leqq \delta$ for all $k \in S$; hence $\beta_{n+1} \leqq \delta^{\omega}<\beta$, a contradiction. Also $\prod_{k<\omega} \alpha_{l(k), k} D \geqq \gamma^{\omega} / D=\gamma^{\omega}=\beta$ so $\prod_{k<\omega} \alpha_{l(k)} / D=\beta$.

Case 2. The cofinality of $\gamma=\omega$. In this case there is a strictly increasing sequence $\left\{\rho_{n}: n<\omega\right\}$ of cardinals whose limit is $\gamma$. Define $l: \omega \rightarrow \omega$ by $l(k)=$ the largest $n \leqq k$ such that $\alpha_{n, k}>\rho_{n}$, or $l(k)=0$ if no such $n$ exists. Then $l$ is not a.e. constant (w.r.t. $D$ ), because otherwise there exists $n<\omega$ and $S \in D$ such that for all $k \in S, \alpha_{n+1, k} \leqq$ $\rho_{n+1}$; hence $\beta_{n+1}=\prod_{k \in S} \alpha_{n+1, k} / D<\beta$, a contradiction. (Note that there are two cases: $\gamma>\omega$, in which case $\beta_{n+1} \leqq \rho_{n+1}^{w}<\beta$, by definition of $\gamma$; or $\gamma=\omega$ in which case $\rho_{n+1}$ is finite, so $\beta_{n+1} \leqq \rho_{n+1}<\beta$.) It follows from Corollary 1.6 (or from 1.3 in case $\gamma=\omega$ ) that $\prod_{k<\omega} \alpha_{l(k), k} / D \geqq$ $\gamma^{\omega}=\beta$.

Let us define $\mathscr{E}$ to be the class of all uniform ultrafilters on any cardinal $\kappa$ which satisfy Theorem 1.7 with $\omega$ replaced by $\kappa$. After seeing an earlier version of this paper, Keisler and Prikry obtained some partial results on the question of whether there exist ultrafilters on uncountable cardinals which are in $\mathscr{E}$. The best result at present, due to Keisler and Prikry [11], is that every good ultrafilter is in $\mathscr{E}$. Their original proof was from the definition of good ultrafilter, which we will not give here (see [1], [2], [9] or [10]); here we give a variation of the argument which uses the fact that if $D$, an ultrafilter on $\kappa$, is good, then, then any ultraproduct with respect to $D$ is $\kappa^{+}$-saturated; (in fact, this property characterizes the good ultrafilters on $\kappa$ : see 
[10]). Since every nonprincipal ultrafilter on $\omega$ is good, the following generalizes Theorem 1.7 above.

Theorem 1.7. (bis). Let $\kappa$ be any cardinal and let $D$ be a good ultrafilter on $\kappa$. Suppose that for each $\nu<\kappa\left\{\alpha_{n, \nu}: n<\omega\right\}$ is a nonincreasing sequence of cardinals. For each $n<\omega$ let $\beta_{n}=\prod_{\nu<\kappa} \alpha_{n, \nu} D$; and let $\beta=\lim _{n \rightarrow \infty} \beta_{n}$. Then there exists $l: \kappa \rightarrow \omega$ such that $l$ is not a.e. constant (w.r.t. $D$ ) and $\Pi_{v<\kappa} \alpha_{l(v), \nu} / D=\beta$.

Proof. As above, we may assume that $\beta_{n}=\beta$ for all $n$ and that $\beta$ is infinite. Define $\gamma$ as in the proof above. There are again two cases:

Case 1. The cofinality of $\gamma>\kappa$. This case is handled in exactly the same way as Case 1 in the proof above.

Case 2. The cofinality of $\gamma \leqq \kappa$. Let $\left\{\rho_{\mu}: \mu<\lambda\right\}$ be a strictly increasing sequence of length $\lambda \leqq \kappa$ of cardinals, whose limit is $\gamma$. For each $\nu<\kappa$ let

$$
U_{\mu}^{(\nu)}=\left\{n: \alpha_{n, \nu}>\rho_{\mu}\right\}
$$

and let

$$
\mathfrak{A}_{\nu}=\left\langle\omega ; U_{\mu}^{(\nu)}\right\rangle_{\mu<\lambda} .
$$

Let $\Gamma=\left\{U_{\mu}(x): \mu<\lambda\right\} \cup\{x \neq \bar{n}: n<\omega\}$, a set of formulas in the language of the $\mathfrak{A}_{\nu}$ 's. We claim that $\Gamma$ is finitely satisfied in $\Pi_{\nu<x} \mathfrak{H}_{\nu} / D=$ $\mathfrak{U}^{*}$. It suffices to prove for each $\mu<\lambda, n<\omega$, that $\left\{U_{\mu}(x), x>n\right\}$ is satisfied in $\mathfrak{R}^{*}$. But then we can take $x$ to be $f_{m} / D$ where $m>n$ and $f_{m}: \kappa \rightarrow \omega$ is the constant function at $m$. Indeed, $f_{m} / D$ satisfies $U_{\mu}(x)$ because otherwise $\left\{\nu<\kappa: m \in U_{\mu}^{(\nu)}\right\} \notin D$; hence $\left\{\nu: \alpha_{m, \nu} \leqq \rho_{\mu}\right\} \in D$ and

$$
\prod_{\nu<\kappa} \alpha_{m, \nu} / D \leqq \rho_{\mu}^{\kappa}<\beta
$$

which is a contradiction. Thus $\Gamma$ is finitely satisfied in $\mathfrak{A}^{*}$, and since $\mathfrak{2}^{*}$ is $\kappa^{+}$-saturated, $\Gamma$ is satisfied by some element $l / D$. Then it follows easily from Corollary 1.6 that $l: \kappa \rightarrow \omega$ is the desired function. This completes the proof.

Keisler has also observed that there are many ultrafilters in $\mathscr{E}$ which are not good. In fact, if $D$ is a uniform ultrafilter on $\kappa>\omega$ and $E$ is a nonprincipal ultrafilter on $\omega$ then $D \otimes E$ is an ultrafilter on $\kappa \times \omega$ (see [1], p. 125 for the definition of $D \otimes E$ ) which is in $\mathscr{E}$ but which is not good. It is apparently an open problem whether every uniform (or regular) ultrafilter is in $\mathscr{E}$.

Our results make use of the assumption of $\omega$-incompleteness of 
the ultrafilter in another essential way besides the use of Lemma 1.2. Indeed we need the following result which is a special case of a more general theorem.

Lemma 1.8. If $D$ is $\omega$-incomplete, then $\prod_{\nu<x} A_{\nu} / D$ is $\omega_{1}$-equationally compact i.e., any countable system of equations with constants from $\Pi_{\nu<x} A_{\nu} / D$ is solvable in $\Pi_{\nu<x} A_{\nu} / D$ iff every finite subsystem is solvable in $\Pi_{\nu<\kappa} A_{\nu} / D$.

Proof. This is a special case of the fact that $\Pi_{\nu<\kappa} A_{\nu} / D$ is $\omega_{1}$ saturated (see [9], §4).

Finally we need the following special case of a more general theorem. Define $\Delta: A \rightarrow A^{\kappa} / D$ by $\Delta(a)=f_{a} / D$ where $f_{a} \in A^{\kappa}$ is the constant function with value $a$.

Lemma 1.9. For any ultrafilter $D, \Delta$ embeds $A$ as a pure subgroup of $A^{\kappa} / D$.

Proof. This is a special case of the fact that $\Delta$ embeds $A$ as an elementary substructure of $A$ ([1], Chapter 5, Lemma 2.3).

§2. Ultrapowers of abelian groups. Our analysis of when an abelian group is an ultrapower begins with Lemma 1.8. If $A$ is an ultrapower with respect to an $\omega$-incomplete ultrafilter, then $A$ is $\omega_{1-}$ equationally compact. Therefore, $A$ is algebraically compact ([5], Exercise 6, p. 162) and we have a structure theorem for $A$ ([5], Proposition 40 or [3], §1):

$$
A \cong \prod_{p} \hat{A}_{p} \oplus A_{d}
$$

where $\hat{A}_{p}$ is the completion in the $p$-adic topology of a direct sum of cyclic $Z_{p}$ modules $\left(Z_{p}=\right.$ valuation ring of $p$-adic valuation of $Q$, the rationals; i.e., $\left.Z_{p}=\{m / n \in Q:(p, n)=1\}\right)$ and $A_{d}$ is the maximal divisible subgroup of $A$. Thus if we let $M^{(\kappa)}$ denote the direct sum of $\kappa$ copies of $M$, we can write

$$
A_{p} \cong \bigoplus_{n} Z\left(p^{n}\right)^{\left(\alpha_{p}, n\right)} \bigoplus Z_{p}^{\left(\beta_{p}\right)}
$$

(where $Z\left(p^{n}\right)=$ cyclic group of order $p^{n}$ ); and

$$
A_{d} \cong \bigoplus_{p} Z\left(p^{\infty}\right)^{\left(\gamma_{p}\right)} \oplus Q^{(\delta)}
$$

(where $Z\left(p^{\infty}\right)$ is the quasicyclic group of type $p^{\infty}([5]$, p. 15)). Hence the algebraically compact group $A$ is completely determined by the cardinals $\alpha_{p, n}, \beta_{p}, \gamma_{p}$, and $\delta$. We sum up some information about these 
cardinals in the following lemma. (Here $m A=\{m x: x \in A\}$ and $m A[n]=$ $\{a \in m A: n a=0\}$; " dim" means dimension as a vector space over $Z(p)$.)

Lemma 2.1. Let $A$ be an algebraically compact group decomposed as in (I), (II) and (III) above; let $T$ be the torsion subgroup of $A$. Then:

(0) $\alpha_{p, n}=\operatorname{dim} p^{n-1} A[p] / p^{n} A[p]$;

(i) (a) $\beta_{p}=\operatorname{dim} A /(T+p A)$;

(b) $\beta_{p} \leqq \lim _{n \rightarrow \infty} \operatorname{dim} p^{n} A / p^{n+1} A$, and equality holds if the righthand side is finite;

(ii) (a) $\gamma_{p}=\operatorname{dim} A_{d}[p]$;

(b) $\gamma_{p} \leqq \lim _{n \rightarrow \infty} \operatorname{dim} p^{n} A[p]$, and equality holds if the righthand side is finite;

(iii) (a) $\delta=\operatorname{rank} A_{d} /\left(T \cap A_{d}\right)$

(b) $\delta \leqq \lim _{n \rightarrow \infty} \operatorname{rank}(n ! A)$.

Proof. Most of these results are readily checked; for (a) see [7], $\S 11$ (the $\alpha_{p, n}$ are the Ulm invariant); for (i)(a) see [7], Lemma 21. Also see [3] for some of these results and for an analysis of the use of the invariants in (0), (i)(b), and (ii)(b) to characterize abelian groups up to elementary equivalence.

The uniformity inherent in the ultrapower construction leads to the fact that the inequalities in the above lemma become equalities in an ultrapower:

LEMMA 2.2. Let $B$ be an abelian group and $D$ an $\omega$-incomplete ultrafilter on a cardinal $\kappa$. Let $A=B^{\kappa} / D$. Then $A$ is algebraically compact, and if $A$ is written as in (I), (II), and (III) above, then for all primes $p$ :

(i ) $\beta_{p}=\lim _{n \rightarrow \infty} \operatorname{dim} p^{n} A / p_{\rightarrow}^{n+1} A$;

(ii) $\gamma_{p}=\lim _{n \rightarrow \infty} \operatorname{dim} p^{n} A[p]$;

(iii) $\delta=\lim _{n \rightarrow \infty} \operatorname{rank}(n ! A)$.

Proof. (i) There is a canonical map

$$
\varphi: p^{n} A / p^{n+1} A \longrightarrow\left(p^{n} B / p^{n+1} B\right)^{\kappa} / D
$$

defined by: $\varphi\left(p^{n}(a / D)+p^{n+1} A\right)=f / D$ where $f(\nu)=p^{n} a(\nu)+p^{n+1} B$, and conversely a map

$$
\psi:\left(p^{n} B / p^{n+1} B\right)^{\kappa} / D \longrightarrow p^{n} A / p^{n+1} A
$$

defined by: $\psi(f / D)=a / D+p^{n+1} A$, where $a(\nu)+p^{n+1} B=f(\nu)$. It is easy to see that $\varphi$ and $\psi$ are inverses of each other, so that

$$
p^{n} A / p^{n+1} A \cong\left(p^{n} B / p^{n+1} B\right)^{\kappa} / D .
$$


Let $\sigma_{p}=\lim _{n \rightarrow \infty} \operatorname{dim} p^{n} A / p^{n+1} A$ and $\sigma_{p}^{\prime}=\lim _{n \rightarrow \infty} \operatorname{dim} p^{n} B / p^{n+1} B . \quad$ By Lemma 2.1(i)(b), $\beta_{p} \leqq \sigma_{p}$ and equality holds if $\sigma_{p}$ is finite, so we may assume $\sigma_{p}$ is infinite. By the above isomorphism, $\sigma_{p}=\left(\sigma_{p}^{\prime}\right)^{k} / D$. On the other hand, we will prove that $\beta_{p} \geqq\left(\sigma_{p}^{\prime}\right)^{\kappa} / D$. Since $D$ is $\omega$-incomplete there is a partition $\kappa=\bigcup_{n<\omega} X_{n}$ such that for all $n, X_{n} \notin D$. Define $q: \kappa \rightarrow \omega$ by: $q(\nu)=n$ if $\nu \in X_{n}$. Then define a function

$$
\theta: \prod_{\nu<\kappa} p^{q(\nu)} B \longrightarrow A / T+p A
$$

( $T=$ torsion subgroup of $A$ ) as follows: if $f \in \prod_{\nu<x} p^{q(\nu)} B$ then $\theta(f)=$ $a / D+(T+p A)$, where $p^{q(\nu)} a(\nu)=f(\nu)$. $\theta$ has the property that if $f, g \in$ $\operatorname{dom} \theta$ and $\left\{\nu: f(\nu) \equiv g(\nu)\left(\bmod p^{q(\nu)+1}\right)\right\} \notin D$, then $\theta(f) \neq \theta(g)$. Therefore $\beta_{p}=\operatorname{Card}(A / T+p A) \geqq \prod_{\nu<k} \operatorname{Card}\left(p^{q(\nu)} B / p^{q(\nu)+1} B\right) / D$ and hence $\beta_{p} \geqq$ $\left(\sigma_{p}^{\prime}\right)^{\kappa} / D$. Hence $\beta_{p}=\sigma_{p}$.

(ii) A similar argument shows that if $\mu_{p}=\lim _{n \rightarrow \infty} \operatorname{dim} p^{n} A[p]$ and $\mu_{p}^{\prime}=\lim _{n \rightarrow \infty} \operatorname{dim} p^{n} B[p]$, then $\gamma_{p}=\mu_{p}=\left(\mu_{p}^{\prime}\right)^{x} / D$. (In this case consider the canonical embedding

$$
\prod_{\nu<\kappa} p^{q(\nu)} B[p] / D \longrightarrow A_{d}[p]
$$

which proves Card $\left(A_{d}[p]\right) \geqq\left(\mu_{p}^{\prime}\right)^{\kappa} / D$.)

(iii) Let $\tau=\lim _{n \rightarrow \infty}(\operatorname{rank}(n ! A))$ and let $\tau^{\prime}=\lim _{n \rightarrow \infty}(\operatorname{Card}(n ! B))$. If $A$ is not of finite exponent, then $\tau^{\prime} \geqq \boldsymbol{\aleph}_{0}$. Since $n ! A \cong(n ! B)^{\kappa} / D$, we see that for sufficiently large $n, \delta \leqq \tau=\operatorname{rank} n ! A=\operatorname{Card}(n ! A)=$ $\left(\tau^{\prime}\right)^{k} / D$-because for uncountable groups, rank $=$ cardinality. Consider the map

$$
\chi: \prod_{\nu<\kappa}(q(\nu) !)^{2} B \longrightarrow A_{d} /\left(T \cap A_{d}\right)
$$

defined as follows: $\chi(f)=a / D+\left(T \cap A_{d}\right)$, where if $f(\nu)=(q(\nu) !)^{2} b_{\nu}$, then $a(\nu)=q(\nu) ! b_{\nu}$. It is easy to check that $\chi$ induces an embedding

$$
\bar{\chi}: \prod_{\nu<\kappa}(q(\nu) !)^{2} B / D \longrightarrow A_{d} /\left(T \cap A_{d}\right)
$$

and therefore, since Card $(n !)^{2} B=\tau^{\prime}$ for sufficiently large $n$ and $\{\nu$ : $q(\nu) \geqq m\} \in D$ for all $m$, we have that $\delta \geqq\left(\tau^{\prime}\right)^{x} / D$. Hence $\delta=\tau=$ $\left(\tau^{\prime}\right)^{x} / D$.

THEOREM 2.3. Let $A$ be an abelian group. The following are equivalent:

(1) There is an $\omega$-incomplete ultrafilter $D$ on a cardinal $\kappa$ and an abelian group $B$ such that $A$ is isomorphic to $B^{r} / D$;

(2) $A$ is algebraically compact, and if $A$ is written as in (I), (II), and (III) above, then for all primes $p$

(i ) $\beta_{p}=\lim _{n \rightarrow \infty} \operatorname{dim} p^{n} A / p^{n+1} A$ 
(ii) $\gamma_{p}=\lim _{n \rightarrow \infty} \operatorname{dim} p^{n} A[p]$

(iii) $\delta=\lim _{n \rightarrow \infty} \operatorname{rank}(n ! A)$

and for all $n<\omega$,

(iv) If $\sigma=\alpha_{p, n}, \beta_{p}$ or $\gamma_{p}$, then either $\sigma$ is finite or $\sigma^{\omega}=\sigma$;

(v) Either $\delta=0$ or $\delta^{\omega}=\delta$;

(3) For all ultrafilters $D$ on $\omega, A$ is isomorphic to $A^{\omega} / D$.

Proof. $\quad(3) \Rightarrow(1)$ is trivial. As for $(1) \Rightarrow(2)$ we have proved (i), (ii), and (iii) in Lemma 2.2; (iv) and (v) follow from Lemma 1.2 and the facts, proved in 2.2, that $\beta_{p}=\left(\sigma_{p}^{\prime}\right)^{\kappa} / D, \gamma_{p}=\left(\mu_{p}^{\prime}\right)^{k} / D, \delta=\left(\tau^{\prime}\right)^{\kappa} / D$, and from the fact that if $\alpha_{p, n}^{\prime}=\operatorname{dim} p^{n-1} B[p] / p^{n} B[p]$, then $\alpha_{p, n}=\left(\alpha_{p, n}^{\prime}\right)^{x} / D$.

(2) $\Rightarrow$ (3) Let $A^{\prime}=A^{\omega} / D$; we will prove that $A^{\prime}$ is isomorphic to $A$. We may assume $D$ is nonprincipal, i.e., $\omega$-incomplete. Then by $1.8, A^{\prime}$ is algebraically compact, so it may be decomposed as in (I), (II), and (III):

$$
\begin{aligned}
& A^{\prime} \cong \prod_{p} \hat{A}_{p}^{\prime} \oplus A_{d}^{\prime} \\
& A_{p}^{\prime} \cong \bigoplus_{n} Z\left(p^{n}\right)^{\left(\alpha_{p}^{\prime}, n^{\prime}\right)} \oplus Z_{p}^{\left(\beta_{p}^{\prime}\right)} \\
& A_{d}^{\prime} \cong \bigoplus_{p} Z\left(p^{\infty}\right)^{\left(\gamma_{p}^{\prime}\right)} \oplus Q^{\left(\hat{\delta}^{\prime}\right)} .
\end{aligned}
$$

By Lemma $1.9, A$ is isomorphic to a pure subgroup of $A^{\prime}$. Hence it follows from Lemma 2.1 that $\alpha_{p, n} \leqq \alpha_{p^{\prime} n}^{\prime}, \beta_{p} \leqq \beta_{p}^{\prime}, \gamma_{p} \leqq \gamma_{p}^{\prime}$ and $\delta \leqq \delta^{\prime}$. We want to prove the opposite inequalities. It is easy to prove that

$$
p^{n-1} A^{\prime}[p] / p^{n} A^{\prime}[p] \cong\left(p^{n-1} A[p] / p^{n} A[p]\right)^{\omega} / D .
$$

Thus by 2.1(0) and 1.1 if $\alpha_{p, n}$ is finite, $\alpha_{p, n}^{\prime}=\alpha_{p, n}$; and if $\alpha_{p, n}$ is infinite, $\alpha_{p, n}^{\prime} \leqq \alpha_{p, n}^{\prime \prime}=\alpha_{p, n}$.

We know that

$$
p^{n} A^{\prime} / p^{n+1} A^{\prime} \cong\left(p^{n} A / p^{n+1} A\right)^{\omega} / D
$$

for all $n$. If $\beta_{p}$ is finite, then by Lemma 2.1(i)(b), $\beta_{p}=\operatorname{dim} p^{n} A / p^{n+1} A$ for sufficiently large $n$, and hence $\beta_{p}^{\prime}=\lim _{n \rightarrow \infty} \operatorname{dim} p^{n} A^{\prime} / p^{n+1} A=\beta_{p}$. If $\beta_{p}$ is infinite, by hypothesis $\beta_{p}=\operatorname{dim} p^{n} A / p^{n+1} A=\operatorname{Card}\left(p^{n} A / p^{n+1} A\right)$ for sufficiently large $n$, and thus by $2.1(\mathrm{i})(\mathrm{b}), \beta_{p}^{\prime} \leqq \lim _{n \rightarrow \infty} \operatorname{Card} p^{n} A^{\prime} / p^{n+1} A^{\prime}=$ $\beta_{p}^{\prime v}=\beta_{p}$.

We know that

$$
p^{n} A^{\prime}[p] \cong\left(p^{n} A[p]\right)^{\omega} / D
$$

for all $n$. If $\gamma_{p}$ is finite, then by 2.1(ii)(b), $\gamma_{p}^{\prime}=\gamma_{p}$.

If $\gamma_{p}$ is infinite, then by hypothesis $\gamma_{p}=$ Card $p^{n} A[p]$ for sufficiently large $n$, so by 2.1(ii)(b) $\gamma_{p}^{\prime} \leqq \lim _{n \rightarrow \infty} \operatorname{Card} p^{n} A[p]=\gamma_{p}^{w}=\gamma_{p}$.

If $\delta$ is finite, then by hypothesis $A$ has finite exponent so $A^{\prime}$ does 
also and thus $\delta^{\prime}=0$. If $\delta$ is infinite, then by hypothesis $\delta=\operatorname{rank}(n ! A)=$ Card $(n ! A)$ for sufficiently large $n$, and thus since

$$
n ! A^{\prime} \cong(n ! A)^{\omega} / D,
$$

we know $\delta^{\prime} \leqq \lim _{n \rightarrow \infty} \operatorname{Card}(n ! A)=\delta^{\omega}=\delta$. The proof of the theorem is complete.

We may generalize Theorem 2.3 as follows:

THEOREM 2.4. Let $A$ be an abelian group, $\kappa$ a cardinal. The following are equivalent:

(1) There is an $(\omega, \kappa)$-regular ultrafilter on a cardinal $\lambda$ and an abelian group $B$ such that $A$ is isomorphic to $B^{\lambda} / D$.

(2) A satisfies 2.3(2)(i), (ii), and (iii) and furthermore:

(iv) $)_{\kappa}$ If $\sigma=\alpha_{p, n}, \beta_{p}$ or $\gamma_{p}$, then either $\sigma$ is finite or $\sigma^{\kappa}=\sigma$;

(v) Either $\delta=0$ or $\delta^{\kappa}=\delta$;

(3) For all $\omega$-incomplete ultrafilters $D$ on $\kappa, A$ is isomorphic to $A^{\kappa} / D$.

Proof. The proof uses Lemma 1.4 to prove 2.4 (iv) ${ }_{\kappa}$ and $(\mathrm{v})_{\kappa}$ and otherwise is completely analogous to the proof of 2.3 .

3. Ultraproducts of abelian groups. We will prove that any ultraproduct of groups with respect to an ultrafilter in $\mathscr{E}$ is an ultrapower. Combined with the results of $\$ 2$ this gives structure theorems for ultraproducts over certain ultrafilters. Using Theorem 1.7 we see that this applies to a large class of ultrafilters.

THEOREM 3.1. For any abelian group $A$ the following are equivalent:

(1) There exists an ultrafilter $D \in \mathscr{E}$ on a cardinal $\kappa$ and there exist groups $B_{\nu}, \nu<\kappa$ such that $A$ is isomorphic to $\Pi_{\nu<\kappa} B_{\nu} / D$;

(2) For all ultrafilters $D$ on $\omega, A$ is isomorphic to $A^{\omega} / D$.

Proof. $(2) \Rightarrow(1)$ is obvious taking into account 1.7. For the converse, assuming (1), it follows from Lemma 1.8 that $A$ is algebraically compact. Thus it suffices to prove that conditions (i)-(v) of Theorem 2.3(2) are satisfied. To prove (i) it suffices to consider the case when $\beta_{p}$ is infinite. Let $\mu_{p}=\lim _{n \rightarrow \infty} \operatorname{dim} p^{n} A / p^{n+1} A$. Then if $\sigma_{n, \nu}=\operatorname{Card} p^{n} B_{\nu} / p^{n+1} B_{\nu}, \mu_{p}=\lim _{n \rightarrow \infty}\left(\Pi_{\nu<\kappa} \sigma_{n, \nu} / D\right)$. By definition of $\mathscr{E}$, there exists a function $l: \kappa \rightarrow \omega$ which is not a.e. constant (w.r.t. $D$ ) such that $\mu_{p}=\Pi_{\nu<\kappa} \sigma_{l(\nu), \nu} / D$. Define a map

$$
\phi: \prod_{\nu<\kappa}\left(p^{l(\nu)} B_{\nu} / p^{l(\nu)+1} B_{\nu}\right) \longrightarrow A /(T+p A)
$$


such that $\phi(f)=a / D+(T+p A)$, where $p^{l(\nu)} a(\nu)+p^{l(\nu)+1} B_{\nu}=f(\nu)$. We claim that $\phi$ induces an injection

$$
\bar{\phi}: \prod_{\nu<\kappa}\left(p^{l(\nu)} B_{\nu} / p^{l(\nu)+1} B_{\nu}\right) / D \longrightarrow A /(T+p A) .
$$

Indeed, if $\phi(f / D)=0$, then there exists $t / D \in T, b / D \in A$ such that

$$
\{\nu \in \kappa: a(\nu)=t(\nu)+p b(\nu)\} \in D \text {. }
$$

Suppose $m(t / D)=0$ where $m=n p^{r},(p, n)=1$. Then since $\{\nu \in \kappa: l(\nu) \geqq$ $r\} \in D$ we have

$$
\left\{\nu \in \kappa: n p^{l(\nu)} a(\nu)=n p^{l(\nu)+1} b(\nu)\right\} \in D
$$

and thus since $(p, n)=1$,

$$
\{\nu \in \kappa: f(\nu)=0\} \in D
$$

i.e., $f / D=0$. Therefore, $\beta_{p}=\operatorname{Card}(A / T+p A) \geqq \mu_{p}$; since the opposite inequality is true by Lemma $2.1(\mathrm{i})(\mathrm{b})$, we have $\beta_{p}=\mu_{p}$. The proofs of 2.3(2)(ii) and (iii) are similar and we will not give them here. For the proof of (iv), consider, for example, $\beta_{p} ; \beta_{p}=\Pi_{\nu<\kappa} \sigma_{n, \nu} / D$ for sufficiently large $n$; if $\sigma_{n, \nu}$ is infinite a.e. (w.r.t. $D$ ) then by $1.2, \beta_{p}^{w}=\beta_{p}$; on the other hand, if $\sigma_{n, \nu}$ is finite a.e. and $\beta_{p}$ is infinite, then $\beta_{p}^{\omega}=$ $\beta_{p}$ by a recent result of Shelah [14]; the proofs of the other cases of (iv), and of (v), are similar. Thus the proof is complete.

Recall that, by 1.7 and 1.7(bis), nonprincipal ultrafilters on $\omega$ and, more generally, good ultrafilters on any cardinal are in $\mathscr{E}$. This leads to the following corollaries:

COROLlaRY 3.2. For any abelian group $A$, the following are equivalent:

(1) There exists a nonprincipal ultrafilter $D$ on $\omega$ and groups $B_{n}$ such that $A$ is isomorphic to $\prod_{n<\omega} B_{n} / D$;

(2) For all ultrafilters $D$ on $\omega, A$ is isomorphic to $A^{\omega} / D$.

CoRollary 3.3. For any abelian group $A$ and cardinal $\kappa>\omega$ consider the following conditions:

(1) There exists a regular ultrafilter $D$ on $\kappa$ which is in $\mathscr{E}$ and groups $\beta_{\nu}$ such that $A$ is isomorphic to $\Pi_{\nu<x} B_{\nu} / D$;

(2) There exists a good ultrafilter $D$ on $\kappa$ and groups $B_{\nu}$ such that $A$ is isomorphic to $\prod_{\nu<\kappa} B_{\nu} / D$;

(3) For all w-incomplete ultrafilters $D$ on $\kappa, A$ is isomorphic to $A^{\kappa} / D$.

Then $(3) \Leftrightarrow(2)$ and $(2) \Longrightarrow(1)$; if $2^{\kappa}=2^{\omega}$ then $(1) \Longrightarrow(3)$. 
Proof of 3.3. (3) $\Rightarrow(2)$ since there exists a good ultrafilter on $\kappa$ [12] and every good ultrafilter is $\omega$-incomplete. $(2) \Rightarrow(1)$ since every good ultrafilter is regular and, by Theorem 1.7 (bis), every good ultrafilter is in $\mathscr{E}$. Assuming (2) we will prove (3), by proving that $A$ satisfies 2.4(2). $A$ satisfies 2.3(2)(i)-(iii) by 3.1 and 1.7 (bis). To prove (iv) ${ }_{\kappa}$ and $(\mathrm{v})_{\kappa}$, consider, for example, $\beta_{p}=\prod_{\nu<\kappa} \sigma_{n, \nu} / D$ (for sufficiently large $n$ : we use the notation of the proof of 3.1). If $\sigma_{n, \nu}$ is infinite a.e. (w.r.t. $D$ ) then $\beta_{p}^{\kappa}=\beta_{p}$ by 1.4. On the other hand, if $\sigma_{n, \nu}$ is finite a.e. and $\beta_{p}$ is infinite it follows from 1.4 that $\beta_{p}=2^{k}$. (For example one can prove that if $D$ is good then there exists $f$ making $D(\omega, \kappa)$ regular such that the power of $f(\nu) \leqq m$ if $m^{m} \leqq$ $\sigma_{n, \nu}<(m+1)^{m+1}$; then take $\alpha_{\nu}=m$ and apply 1.4.) The proofs of the other parts of (iv) $)_{\kappa}$ and $(\mathrm{v})_{\kappa}$ are similar.

Finally, assuming (1) and $2^{\kappa}=2^{\omega}$ we prove (3). The prove is the same as that above except in the case where $\beta_{p}$ (or $\alpha_{p, n}$ or $\gamma_{p}$ or $\delta$ ) is finite. In that case we conclude $\beta_{p}=2^{\kappa}$ because by $1.3 \beta_{p} \geqq 2^{\omega}=2^{\kappa}$. This completes the proof of 3.3 .

In contrast to 3.1 and 3.3 we have the following:

THEOREM 3.4. (a) If $2^{\kappa}>2^{\omega}$, there is a regular ultrafilter $D$ on $\kappa$ which is in $\mathscr{E}$ and groups $B_{\nu}$ such that $\Pi_{\nu>\kappa} B_{\nu} / D$ is not isomorphic to $A^{\kappa} / D^{\prime}$ for any group $A$ and any regular ultrafilter $D^{\prime}$ on $\kappa$.

(b) If there is a regular ultrafilter $D$ on $\kappa$ which is not in $\mathscr{E}$ then there exist groups $B_{\nu}$ such that $\Pi_{\nu<x} B_{\nu} / D$ is not an ultrapower with respect to any $\omega$-incomplete ultrafilter.

Proof. (a) We identify $\kappa$ with $\kappa \times \omega$ and let $D$ be any ultrafilter on $\kappa \times \omega$ of the form $E \otimes F$ where $E$ is a regular ultrafilter on $\kappa$ and $F$ is a nonprincipal ultrafilter on $\omega$ (see [1] for the definition of $E \otimes F)$. It is readily checked that $D$ is regular and in $\mathscr{E}$. For $(\nu, n) \in \kappa \times \omega$ let $\rho_{\nu, n}=n$ and define

$$
B_{\nu, n}=Z\left(p^{\infty}\right)^{\left(\rho_{\nu}, n\right)} \oplus Q
$$

for some fixed prime $p$. If $C=\prod_{\kappa \times \omega} B_{\nu, n} / D$, then for all $m$

$$
\gamma_{p}=\operatorname{dim} p^{m} C[p]=\prod_{\kappa \times \omega} \rho_{\nu, n} / D=\prod_{n<\omega}\left(\prod_{\nu<\kappa} \rho_{\nu, n} / E\right) / F=\prod_{n<\omega} n / F=2^{\omega}<2^{\kappa} .
$$

Hence $\gamma_{p}^{\kappa} \neq \gamma_{p}$ so $C$ does not satisfy $2.4(2)(\mathrm{iv})_{\kappa}$.

(b) Let $D$ be a regular ultrafilter on a cardinal $\kappa$ such that there exist nonincreasing sequences of cardinals $\left\{\sigma_{n, \nu}: n<\omega\right\}$ such that if $l: \kappa \rightarrow \omega$ satisfies

$$
\prod_{\nu<\kappa} \sigma_{l(\nu), \nu} / D=\lim _{n \rightarrow \infty}\left(\prod_{\nu<\kappa} \sigma_{n, \nu} / D\right)
$$

then $l$ is a.e. constant with respect to $D$. Fix a prime $p$ and let 


$$
B_{\nu}=\bigoplus_{1 \leqq m<\omega} Z\left(p^{m}\right)^{\left(\alpha_{m}, \nu\right)} \oplus Z\left(p^{\infty}\right)^{\left(\beta_{\nu}\right)}
$$

where the exponents are chosen such that for all $\nu$ and $n$,

$$
\left(\sum_{m \geqq n+1} \alpha_{m, \nu}\right)+\beta_{\nu}=\sigma_{n, \nu}
$$

i.e., $\operatorname{dim} p^{n} B_{\nu}[p]=\sigma_{n, \nu}$ (This is possible because $\left\{\sigma_{\nu, \kappa}: n<\omega\right\}$ is nonincreasing.) Let $C=\prod_{\nu<\kappa} B_{\nu} / D$. We claim that $\operatorname{dim} C_{d}[p] \varsubsetneqq$ $\lim _{n \rightarrow \infty} \operatorname{dim} p^{n} C[p]$ which implies $C$ is not an ultrapower. Let $\tau=$ $\lim _{n \rightarrow \infty} \operatorname{dim} p^{n} C[p]=\lim _{n \rightarrow \infty}\left(\Pi_{\nu<x} \sigma_{n, \nu} / D\right) ; \tau \geqq 2^{\kappa}$ because $D$ is regular. And $\tau>2^{\kappa}$ because otherwise we are in Case 2 of Theorem $1.7(\gamma=$ $\omega)$ and the same proof works. For any $f / D \in C_{d}[p]$, if $q: \kappa \rightarrow \omega$ is defined as in the proof of 2.2 , define $l_{f}: \kappa \rightarrow \omega$ by $l_{f}(\nu)=$ largest $m \leqq q(m)$ such that $p^{m} \mid f(\nu)$. Suppose $\operatorname{dim} C_{d}[p]=\tau$; then there is an $l \in \omega^{x}$ such that $l_{f}=l$ for $\tau$ elements $f / D \in C_{d}[p]$. Then $l$ is not a.e. constant and

$$
\operatorname{dim} \prod_{\nu<\kappa} p^{l(\nu)} B_{\nu}[p] / D=\tau
$$

Hence $\Pi_{\nu<\kappa} \sigma_{l(\nu), \nu} / D=\tau$, which is a contradiction.

\section{REFERENCES}

1. J. L. Bell and A. B. Slomson, Models and Ultraproducts: An Introduction, NorthHolland, Amsterdam, 1969)

2. C. C. Chang, Ultraproducts and Other Methods of Constructing Models, in Sets, Models and Recursion Theory, ed. by J.N. Crossley, North-Holland, Amsterdam, 1967, 85-121.

3. P. C. Eklof and E. R. Fisher, The elementary theory of abelian groups, to appear in Annals of Math. Logic, 4 (1972), 115-171.

4. T. Frayne, A. Morel, and D. Scott, Reduced direct products, Fund. Math., 51 (1962), 195-228.

5. L. Fuchs, Infinite Abelian Groups, vol. I, Academic Press, New York, 1970.

6. R. Jensen, to appear.

7. I. Kaplansky, In finite Abelian Groups, rev. ed., University of Mich. Press, Ann Arbor, 1969.

8. H. J. Keisler, On cardinalities of ultraproducts, Bull. Amer. Math. Soc., 70 (1964), 644-647.

9. - A Survey of Ultraproducts, in Logic, Methodology and the Philosophy of Science, ed. by Y. Bar-Hillel, North-Holland, Amsterdam, 1965, 112-126.

10. - Ultraproducts and saturated models, Indag. Math, 26 (1964), 178-186.

11. H. J. Keisler and K. Prikry, to appear.

12. K. Kunen, Ultrafilters and independent sets, to appear.

13. K. Prikry, On a problem of Gillman and Keisler, Annals of Math Logic, 2 (1970), 179-187.

14. S. Shelah, On the cardinality of ultraproducts of finite sets, J. Symbolic Logic, 35 (1970), 83-84.

15. J. Shoenfield, Measurable Cardinals, in Logic Colloquium '69 ed. by R. Gandy and C.M.E. Yates, North-Holland, Amsterdam, 1970, 19-49.

Received April 18, 1972. Research supported by NSF GP-29254. 
